\title{
ON HASSE ZETA FUNCTIONS OF ENVELOPING ALGEBRAS OF SOLVABLE LIE ALGEBRAS
}

\author{
TAKAKo FuKaYa
}

\section{Introduction}

In the paper [F1], we generalized the Hasse zeta functions $\zeta_{A}(s)$ of commutative finitely generated rings $A$ over the ring $Z$ of integers, to noncommutative rings.

The aim of this paper is to prove

THEOREM 1.1. Let $R$ be a finitely generated commutative ring over $Z$, let $\mathfrak{g}$ be a solvable Lie algebra over $R$ which is free of finite rank $n$ as an $R$-module, and let $A$ be the universal enveloping algebra of $\mathfrak{g}$ over $R$. Then

$$
\zeta_{A}(s)=\zeta_{R}(s-n)
$$

1.2. We review the definition of the function $\zeta_{A}(s)$. For a (not necessarily commutative) finitely generated $\operatorname{ring} A$ over $Z$, the Hasse zeta function $\zeta_{A}(s)$ of $A$ is defined by

$$
\zeta_{A}(s)=\prod_{r \geq 1} \zeta_{A, r}(s)
$$

where $r$ runs over integers $\geq 1$, and

$$
\zeta_{A, r}(s)=\prod_{p} \exp \sum_{n=1}^{\infty} \frac{\sharp \Im_{A, r}\left(\boldsymbol{F}_{p^{n}}\right)}{n}\left(p^{-s}\right)^{n}
$$

where $\mathfrak{S}_{A, r}$ is a certain scheme of finite type over $\boldsymbol{Z}, p$ runs over prime numbers, and $\boldsymbol{F}_{p^{n}}$ is a finite field with $p^{n}$ elements, so the function $\zeta_{A, r}(s)$ coincides with the product of Weil's zeta functions of $\mathfrak{S}_{A, r} \otimes{ }_{Z} \boldsymbol{F}_{p}$ [We] for all prime numbers $p$. For the algebraic closure $K$ of $\boldsymbol{F}_{p}, \mathfrak{S}_{A, r}(K)$ is identified with the set of the isomorphism classes of all $r$-dimensional irreducible representations of $A$ over $K$, and $\mathfrak{\Xi}_{A, r}\left(\boldsymbol{F}_{p^{n}}\right)$ is identified with the $\operatorname{Gal}\left(K / \boldsymbol{F}_{p^{n}}\right)$-fixed part of $\mathfrak{\Xi}_{A, r}(K)$.

Theorem 1.1 is deduced from the following Theorem 1.3.

THEOREM 1.3. Let $B$ be a finitely generated algebra over $Z$, let $\delta$ be a derivation of $B$, and let $A$ be the ring $\left\{\sum_{l=0}^{N} b_{i} t^{l} ; N \geq 0, b_{i} \in B\right\}$ in which $t$ is an indeterminate and the multiplication is expressed as $t b-b t=\delta(b)(b \in B)$. Then

$$
\zeta_{A}(s)=\zeta_{B}(s-1)
$$


We show that Theorem 1.1 follows from Theorem 1.3.

We may assume that $R$ is a finite field of characteristic $p>0$, for $\zeta_{R}(s), \zeta_{A}(s)$ are products of $\zeta_{R / \mathfrak{m}}(s), \zeta_{A / \mathfrak{m} A}(s)$ over all maximal ideals $\mathrm{m}$ of $R$, respectively. So assume $R$ is a finite field $k$.

Since $\mathfrak{g}$ is a solvable Lie algebra, there exists a sequence of subalgebras of $\mathfrak{g}$

$$
\mathfrak{g}=\mathfrak{g}_{0} \supset \mathfrak{g}_{1} \supset \cdots \supset \mathfrak{g}_{n}=\{0\}
$$

where $\mathfrak{g}_{i}$ is of dimension $n-i$ as a $k$-vector space, and $\left[\mathfrak{g}_{i-1}, \mathfrak{g}_{i}\right] \subset \mathfrak{g}_{i}$ for $1 \leq i \leq n$. Take the universal enveloping algebras of $\mathfrak{g}_{i-1}$ and $\mathfrak{g}_{i}$ as $A$ and $B$, respectively, and apply Theorem 1.3 inductively, then we obtain Theorem 1.1.

In section 2, we prove Theorem 1.3.

A proof of a special case of Theorem 1.1 and a proof of Theorem 1.3 in the case $B$ is commutative are given in our previous papers [F2], [F3], respectively.

I am very grateful to Professor Kazuya Kato who suggested me to study this subject, gave me much essential advice and encouragement.

\section{Proof of Theorem 1.3}

2.1. Let $A, B$ and $\delta$ be as in Theorem 1.3. Since $\zeta_{A}(s)=\prod_{p} \zeta_{A / p A}(s)$, $\zeta_{B}(s)=\prod_{p} \zeta_{B / p B}(s)$ where $p$ ranges over all prime numbers, we may assume that $B$ is an $\boldsymbol{F}_{p}$-algebra. Let $k=\boldsymbol{F}_{p}$, and let $K$ be the algebraic closure of $k$. Let $\mathfrak{S}_{A}=\coprod_{r \geq 1} \mathfrak{\Xi}_{A, r}$, and for an extension $k^{\prime}$ of $k$, let $\mathfrak{\Xi}_{A}\left(k^{\prime}\right)$ be the set of $k^{\prime}$-rational points of $\mathfrak{S}_{A}$. We define $\mathfrak{\Xi}_{B}$ and $\mathfrak{\Xi}_{B}\left(k^{\prime}\right)$ as in the case of $A$. Let $B_{K}=$ $B \otimes_{k} K$.

Let $M$ be a finite dimensional irreducible representation of $A$ over $K$, and let $N$ be an irreducible representation of $B$ over $K$ which is a subrepresentation of $M$. Let $\chi_{N}: B_{K} \rightarrow \operatorname{End}_{K}(N)$ be the action of $B_{K}$ on $N$.

DefinITION 2.2. Let $\left\{\delta_{j} ; 1 \leq j \leq m\right\}(m \in Z, m \geq 1)$ be a family of $k$-derivations of $B$. We say " $\left\{\chi_{N} \circ \delta_{j} ; 1 \leq j \leq m\right\}$ are linearly independent (resp. dependent) modulo inner derivation" if the canonical images of $\left\{\chi_{N} \circ \delta_{j} ; 1 \leq j \leq m\right\}$ in the space

$$
\left\{B_{K} \rightarrow \operatorname{End}_{K}(N) ; K \text {-linear }\right\} /\left\{B_{K} \rightarrow \operatorname{End}_{K}(N) ; b \mapsto \chi_{N}\left(\left[b, b_{0}\right]\right) \text { for some } b_{0} \in B_{K}\right\}
$$

are linearly independent (resp. dependent) over $K$.

LEMma 2.3. There exists an integer $l \geq 0$ such that $\left\{\chi_{N} \circ \delta^{p^{l}} ; 0 \leq i \leq l-1\right\}$ are linearly independent modulo inner derivation and $\left\{\chi_{N} \circ \delta^{p^{l}} ; 0 \leq i \leq l\right\}$ are linearly dependent modulo inner derivation.

Proof. Note that $\delta^{p^{t}}(b)$ is a $k$-derivation for any $i \in Z, i \geq 0$ ([S-F] Chapter 1, Proposition 2.3.2). In the $K$-linear space

$$
\left\{B_{K} \rightarrow \operatorname{End}_{K}(N) ; K \text {-linear }\right\} /\left\{B_{K} \rightarrow \operatorname{End}_{K}(N) ; b \mapsto \chi_{N}\left(\left[b, b_{0}\right]\right) \text { for some } b_{0} \in B_{K}\right\}
$$


the images of $\left\{\chi_{N} \circ \delta^{p^{m}} ; m \in Z, m \geq 0\right\}$ are contained in the following $K$-linear subspace: $\left\{h: B_{K} \rightarrow \operatorname{End}_{K}(N) ; K\right.$-linear, $\left.h(a b)=\chi_{N}(a) h(b)+h(a) \chi_{N}(b)\right\} /$ $\left\{B_{K} \rightarrow \operatorname{End}_{K}(N) ; b \mapsto \chi_{N}\left(\left[b, b_{0}\right]\right)\right.$ for some $\left.b_{0} \in B_{K}\right\}$, which is finite dimensional over $K$ since the maps $h$ satisfying the condition are determined by the values of $h$ at the generators of $B$ over $k$.

We use the same notation $\delta$ for the $K$-derivation of $B_{K}$ which is induced from $\delta$ in Theorem 1.3.

We have the following proposition.

Proposition 2.4. Let $l$ be as in Lemma 2.3. Then the map

$$
N^{\oplus p^{l}} \rightarrow M ;\left(x_{\imath}\right)_{0 \leq l \leq p^{l}-1} \mapsto \sum_{l=0}^{p^{l}-1} t^{l} x_{l}
$$

is bijective.

We prove Proposition 2.4 by using the following Lemmas 2.5, 2.6, and 2.7.

LEMMA 2.5. For $b \in B_{K}$ and for $m \in \boldsymbol{Z}, m \geq 0$,

$$
b t^{m}=\sum_{j=0}^{m}(-1)^{J}\left(\begin{array}{c}
m \\
j
\end{array}\right) t^{m-j} \delta^{J}(b) .
$$

Especially, let $f:\{a \in Z ; a>0\} \rightarrow\{a \in Z ; a>0\}$ be the function defined by $f(a)=a-p^{r}$ where $p^{r} \| a$. For an integer $m>0$, for any $b \in B_{K}$, and for $x \in N$,

$$
\left(t^{m} b-b t^{m}\right) x=\alpha t^{f(m)} \delta^{m-f(m)}(b) x
$$

$+\left(\right.$ a linear combination of the elements $\left.t^{l} \delta^{m-l}(b) x(0 \leq i<f(m))\right)$

where $\alpha \in \boldsymbol{F}_{p}, \alpha \neq 0$.

Proof. See [S-F] Chapter 1, Proposition 1.3.

Lemma 2.6. Assume that $\left\{\chi_{N} \circ \delta^{p^{l}} ; 0 \leq i \leq l-1\right\}$ are linearly independent over $K$ modulo inner derivation. Then the map

is injective.

$$
N^{\oplus p^{\prime}} \rightarrow M ; \quad\left(x_{t}\right)_{0 \leq \imath \leq p^{l}-1} \mapsto \sum_{t=0}^{p^{l}-1} t^{l} x_{t}
$$

Proof. We prove this by induction. Let $i$ be an integer such that $1 \leq i \leq$ $p^{l}-1$, and assume that

$$
N+t N+\cdots+t^{l-1} N \cong N^{\oplus \imath}
$$

as $K$-linear spaces by the map defined above. We show that

$$
N+t N+\cdots+t^{l-1} N+t^{l} N \cong N^{\oplus(i+1)} \text {. }
$$


This (i) is equivalent to the fact that the map

$$
t^{l}: N \rightarrow M /\left(N+t N+\cdots+t^{l-1} N\right) ; x \mapsto t^{l} x \bmod N+t N+\cdots+t^{l-1} N
$$

is injective.

Now we have a lemma.

LEMMA 2.6.1. The above map $t^{l}$ is a B-homomorphism.

Proof. This follows from Lemma 2.5.

By Lemma 2.6.1, if the map $t^{l}$ is not injective, it is the 0-map (since $N$ is irreducible). We assume that $t^{l}$ is the 0 -map, and will get a contradiction.

The fact that $t^{l}$ is the 0 -map is equivalent to

$$
t^{l} N \subset N+t N+\cdots+t^{l-1} N .
$$

Then for $x \in N$, it can be expressed as

$$
t^{l} x=g_{0}(x)+\cdots+t^{l-1} g_{i-1}(x)
$$

where $g_{j}: N \rightarrow N$ is a $K$-linear map for $0 \leq j \leq i-1$. For $b \in B_{K}$, by Lemma 2.5 ,

$$
\begin{aligned}
b t^{l} x & =\sum_{j=0}^{l}(-1)^{J}\left(\begin{array}{l}
i \\
j
\end{array}\right) t^{l-J} \delta^{J}(b) x=t^{l} b x+\sum_{j=1}^{l}(-1)^{J}\left(\begin{array}{c}
i \\
j
\end{array}\right) t^{l-J} \delta^{J}(b) x \\
& =g_{0}(b x)+\cdots+t^{l-1} g_{i-1}(b x)+\sum_{j=1}^{l}(-1)^{J}\left(\begin{array}{c}
i \\
j
\end{array}\right) t^{l-J} \delta^{J}(b) x .
\end{aligned}
$$

Moreover,

$$
b t^{l} x=b g_{0}(x)+\cdots+b t^{l-1} g_{i-1}(x) .
$$

We compare the two equations (ii) and (iii). The most important parts in (ii) and (iii) are the $t^{f(i)} N$-components where $f$ is as in Lemma 2.5 .

To prepare to compare the equations, we have some lemmas.

Lemma 2.6.2. We have the following equation. For $b \in B_{K}$ and for $m \in Z$, $0 \leq m \leq i-1$,

(iv) $g_{m}(b x)+(-1)^{l-m}\left(\begin{array}{c}i \\ m\end{array}\right) \delta^{l-m}(b) x=b g_{m}(x)+\sum_{j=1}^{l-1-m}(-1)^{J}\left(\begin{array}{c}m+j \\ m\end{array}\right) \delta^{J}(b) g_{m+j}(x)$.

Proof. The left hand side is the $t^{m} N$-component in $b t^{l} x$ in the equation (ii), and the right hand side is that in (iii). 
LEMMA 2.6.3. (1) For $j(0 \leq j \leq i-1)$ such that $f(j) \geq f(i)$, $g_{j}$ is a scalar map. That is, $g_{j}(x)=C_{j} x(x \in N)$ for some $C_{J} \in K$.

(2) For $j(0 \leq j \leq i-1)$ such that $f(j)>f(i), g_{j}$ is the 0-map.

Proof. We fix an integer $m$ such that $f(i)<m \leq i-1$. Assume that for $j(0 \leq j \leq i-1)$ such that $j>m, g_{j}$ is a scalar map $C_{J}\left(C_{J} \in K\right)$, and for $j(0 \leq j \leq i-1)$ such that $f(j)>m, g_{j}$ is the 0 -map.

We show that $g_{m}$ is a scalar map $C_{m}\left(C_{m} \in K\right)$, and for $j(0 \leq j \leq i-1)$ such that $f(j)=m, g_{j}$ is the 0-map.

Remark that $j>f(j)$, so Lemma 2.6 .3 follows from this by downward induction on $m$.

We consider Lemma 2.6.2. Since $m>f(i)$, from the computation of the coefficient, the part $(-1)^{i-m}\left(\begin{array}{c}c \\ m\end{array}\right) \delta^{l-m}(b) x$ in the left hand side of the equation (iv) is 0 . So the equation (iv) is

$$
g_{m}(b x)=b g_{m}(x)+\sum_{j=1}^{l-1-m}(-1)^{J}\left(\begin{array}{c}
m+j \\
m
\end{array}\right) \delta^{J}(b) g_{m+j}(x) .
$$

By the theorem of Burnside ([F-D] Corollary 1.16), any $K$-linear map: $N \rightarrow N$ is obtained as an action of an element of $B_{K}$. So we write $g_{j}(x)=b_{j} x$ for $b_{j} \in B_{K} \quad(0 \leq j \leq i-1)$. By the hypothesis of this induction, the equation (v) is equivalent to the equation

$$
\left[b_{m}, b\right] x=\sum_{\substack{j \in f^{-1}(m) \\ 0 \leq J \leq l-1}} \alpha_{j} C_{j} \delta^{j-m}(b) x
$$

where $\alpha_{j} \in \boldsymbol{F}_{p}, \alpha_{j} \neq 0$ (We denote $m+j$ in (v) by $j$ here). For $j \in f^{-1}(m)$ such that $0 \leq j \leq i-1, j-m=p^{r}$ for some $r \in Z, 0 \leq r \leq l-1$. From the linear independence of $\left\{\chi_{N} \circ \delta^{p^{i}} ; 0 \leq i \leq l-1\right\}$ modulo inner derivation, $C_{J}=g_{j}=0$ for $j \in f^{-1}(m)$ such that $0 \leq j \leq i-1$. So $\chi_{N}\left(b_{m} b\right)-\chi_{N}\left(b b_{m}\right)=0$. Hence $g_{m}=$ $\chi_{N}\left(b_{m}\right)$ is $B$-linear. Since $N$ is irreducible, $g_{m}$ is a scalar map.

Now we accomplish the proof of Lemma 2.6.

We compare the $t^{f(i)} N$-components in (ii) and (iii). We put $m=f(i)$ in (iv). The coefficient

$$
(-1)^{l-f(i)}\left(\begin{array}{c}
i \\
f(i)
\end{array}\right)
$$

which is on the left hand side of (iv) is not zero. By Lemma 2.6.3 and the argument in its proof,

$$
\chi_{N}\left(b_{f(i)} b\right)-\chi_{N}\left(b b_{f(i)}\right)=\alpha_{i} \chi_{N} \circ \delta^{l-f(i)}(b)+\sum_{\substack{j \in f^{-1}(f(i)) \\ 0 \leq J \leq l-1}} \alpha_{j} C_{J} \chi_{N} \circ \delta^{J-f(i)}(b)
$$


where $\alpha_{j} \in \boldsymbol{F}_{p}, \alpha_{j} \neq 0\left(j \in f^{-1}(f(i)), 0 \leq j \leq i\right)$. For each $j \in f^{-1}(f(i))$ such that $0 \leq j \leq i$, there exists $r \in Z, 0 \leq r \leq l-1$ such that $j-f(i)=p^{r}$. This contradicts the assumption that $\left\{\chi_{N} \circ \delta^{p^{l}} ; 0 \leq i \leq l-1\right\}$ are linearly independent modulo inner derivation.

LEMMA 2.7. Assume that $\left\{\chi_{N} \circ \delta^{p^{l}} ; 0 \leq i \leq l-1\right\}$ are linearly independent and $\left\{\chi_{N} \circ \delta^{p^{i}} ; 0 \leq i \leq l\right\}$ are linearly dependent modulo inner derivation. Then there exists an irreducible representation $N^{\prime}$ of $B$ over $K$ which is a subrepresentation of $M$ such that $N^{\prime} \cong N$ as a $B_{K}$-module and

$$
\sum_{l=0}^{p^{l}-1} t^{l} N^{\prime}=M
$$

Proof. Assume that

$$
\chi_{N} \circ \delta^{p^{l}}=\sum_{i=0}^{l-1} \gamma_{i} \chi_{N} \circ \delta^{p^{l}}+\chi_{N} \circ\left[b_{0}, \quad\right]
$$

where $\gamma_{i} \in K, b_{0} \in B_{K}$. Put

$$
t^{\prime}=t^{p^{l}}-\sum_{l=0}^{l-1} \gamma_{i} t^{p^{t}}-b_{0} .
$$

Since $\left[t^{p^{i}}, \quad\right]=\delta^{p^{l}}, \chi_{N}\left(b t^{\prime}-t^{\prime} b\right)=0$ for all $b \in B_{K}$. Let $W=\{x \in M ; b x=0$ for any $b \in \operatorname{Ann}(N)\}$, where $\operatorname{Ann}(N)=\left\{b \in B_{K} ; b N=0\right\}$. Then $W$ is stable under the actions of $B_{K}$ and $t^{\prime}$. Let $N^{\prime}$ be the irreducible representation of $B\left[t^{\prime}\right]$ over $K$ which is contained in $W$. In $N^{\prime}$, the action of $t^{\prime}$ commutes with the actions of $B\left[t^{\prime}\right]$, and hence is a scalar map. So $N^{\prime}$ is an irreducible representation also of $B_{K}$. Since $\operatorname{Ann}(N)$ kills $N^{\prime}, N^{\prime}$ is isomorphic to $N$ over $B_{K}$. The subrepresentation $\sum_{l=0}^{p^{l}-1} t^{l} N^{\prime}$ of $M$ is stable under the actions of the elements of $B_{K}$ and $t$. So it coincides with $M$. Hence we obtain the result.

LEMMA 2.8. Let $N^{\prime \prime}$ be an irreducible representation of $B$ over $K$ which is contained in $M$. Then $N^{\prime \prime}=N$.

Proof. As a $B_{K}$-module, $M$ has a composition series whose all quotients are isomorphic to $N$. Hence $N^{\prime \prime} \cong N$ over $B_{K}$.

To prove $N^{\prime \prime}=N$, it is sufficient to prove that the image of any $B_{K^{-}}$ homomorphism

$$
h: N \rightarrow M=\sum_{l=0}^{p^{l}-1} t^{l} N
$$


is contained in $N$. Write $h(x)=\sum_{l=0}^{p^{l}-1} t^{l} h_{i}(x)(x \in N)$ where $h_{i}\left(0 \leq i \leq p^{l}-1\right)$ are $K$-linear maps $N \rightarrow N$. For any $x \in N$ and $b \in B_{K}$,

and

$$
h(b x)=h_{0}(b x)+t h_{1}(b x)+\cdots+t^{p^{l}-1} h_{p^{l}-1}(b x),
$$

$$
h(b x)=b h_{0}(x)+b t h_{1}(x)+\cdots+b t^{p^{l}-1} h_{p^{l}-1}(x) .
$$

We compare the $t^{m} N$-components $\left(0 \leq m \leq p^{l}-1\right)$ of the above two equations, then we have

$$
h_{m}(b x)=b h_{m}(x)+\sum_{j=1}^{p^{\prime}-1-m}(-1)^{J}\left(\begin{array}{c}
m+j \\
m
\end{array}\right) \delta^{J}(b) h_{m+j}(x) .
$$

This equation has the same form as (v). So from the argument in the proof of Lemma $2.6 .3, h_{i}=0$ for $1 \leq i \leq p^{l}-1$. So $h(N) \subset N$.

From Lemma 2.8, we obtain

COROllary 2.8.1. There exists a surjective map

$$
\pi: \mathfrak{\Im}_{A}(K) \rightarrow \mathfrak{\Im}_{B}(K) \text {; the class of } M \mapsto \text { the class of } N \text {. }
$$

This map $\pi$ commutes with the action of the Galois group $\operatorname{Gal}(K / k)$.

By Lemmas 2.6, 2.7, and 2.8, we obtain Proposition 2.4.

2.9. Let $l$ be as in Lemma 2.3.

From the above argument, we have that the irreducible representation $M$ of $A$ over $K$ is determined by $\chi_{N}$ and the action of $t^{p^{l}}$. Write

$$
\chi_{N} \circ \delta^{p^{l}}=\sum_{l=0}^{l-1} \gamma_{i} \chi_{N} \circ \delta^{p^{l}}+\left[b_{0}, \quad\right]
$$

where $\gamma_{i} \in K$ and $b_{0} \in B_{K}$. Put

$$
t^{\prime}=t^{p^{l}}-\sum_{l=0}^{l-1} \gamma_{i} t^{p^{l}}-b_{0}
$$

By Proposition 2.4, the action of $t^{\prime}$ on $M$ is completely determined by its action on $N$, and from the argument of the proof of Lemma 2.7, $t^{\prime}$ acts on $N$ as a scalar. Then we have for $x \in N$,

$$
t^{\prime} x=c x
$$

for some $c \in K$. Hence

$$
t^{p^{l}} x=\sum_{l=0}^{l-1} \gamma_{i} t^{p^{l}} x-\left(b_{0}+c\right) x
$$

We can take $c \in K$ arbitrarily. 
From this and Corollary 2.8.1, for each finite extension $\boldsymbol{F}_{q}$ of $k$ which has $q$ elements and $x \in \mathfrak{S}_{B}\left(\boldsymbol{F}_{q}\right)$, the $\operatorname{Gal}\left(K / \boldsymbol{F}_{q}\right)$-set $\pi^{-1}(x)$ is a $K$-principal homogeneous space. Since $H^{1}\left(\operatorname{Gal}\left(K / \boldsymbol{F}_{q}\right), K\right)=\{0\}, \pi^{-1}(x)$ is isomorphic to $K$ as a $\mathrm{Gal}$ $\left(K / \boldsymbol{F}_{q}\right)$-set. Hence we have

$$
\sharp \Im_{A}\left(\boldsymbol{F}_{q}\right)=\sharp \Im_{B}\left(\boldsymbol{F}_{q}\right) \cdot q .
$$

This proves Theorem 1.3.

\section{Remark}

For a solvable Lie algebra $g$ over $R$ where $R$ is a finitely generated commutative ring over $Z$, we have Theorem 1.1 which says that the Hasse zeta function of the universal enveloping algebra of $\mathrm{g}$ over $R$ is determined only by its rank over $R$.

But when Lie algebra $\mathfrak{g}$ is not solvable, we cannot say such things. For example, if $A$ is the universal enveloping algebra of $s l_{2}(\boldsymbol{Z})$, we have

$$
\zeta_{A}(s)=\zeta(s-3) \prod_{p: \text { odd prime }}\left(1-p^{-(s-1)}\right)^{(p-1) / 2} \prod_{p: \text { odd prime }}\left(1-p^{-s}\right)^{-(p-1) / 2}
$$

(see $[\mathrm{F} 1])$.

\section{REFERENCES}

[F-D] B. FARb AND R. K. Dennis, Noncommutative Algebra, Grad. Texts in Math., 144, Springer-Verlag, 1993.

[S-F] H. Strade AND R. Farnsteiner, Modular Lie Algebras and their Representations, Monogr. Textbooks Pure Appl. Math., 116, Marcel Dekker, New York, 1988.

[We] A. WeIL, Numbers of solutions of equations over finite fields, Bull. Amer. Math. Soc., 55 (1949), pp. 497-508.

[F1] T. FuKAYA, Hasse zeta functions of non-commutative rings, J. Algebra, 208 (1998), pp. 304342.

[F2] T. FukAYA, On Hasse zeta functions of enveloping algebras of solvable Lie algebras, Proc. Japan Acad. Ser. A Math. Sc1., 72 (1996), pp. 187-188.

[F3] T. FukAYA, On Hasse zeta functions of enveloping algebras of solvable Lie algebras 2, Proc. Japan Acad. Ser. A Math. Sc1., 72 (1996), pp. 199-201.

Graduate School of Mathematical Sciences

THE UNIVERSITY OF TOKYO

3-8-1 Komaba, Meguro-Ku

ТОКYO, 153-8914

JAPAN 
